\title{
FORUM
}

\section{The Metrication of Navigation}

\section{Ronald Turner}

INTRODuction. Since government and industry have officially accepted the introduction of the International System (S.I.) Units for all forms of measurement it is relevant to examine the implications of this for marine navigation.

The PRESENT situation. Marine navigation is no different from many other fields in that a proliferation of units is used to measure the same dimension and, moreover, these units have no systematic relationship to each other. In this respect length is the worst offender: we measure heights in feet, depths of water in fathoms, distances in cables or nautical miles and moreover these nautical miles may be British standard, International standard or those precisely determined by latitude.

This proliferation of units can and does cause confusion and unnecessarily increases the demands made on the newcomer to navigation whether he be the young man starting his career or the engineer, mathematician or scientist who finds that his interests have led him into navigation.

Current Developments. The Hydrographic Department has decided to alter drastically the manner in which information is presented on charts with the aim of making them more legible and more suitable for current requirements. The introduction of these new charts will be a long process extending over many years and it is therefore necessary to consider carefully what alterations should be made. It has been decided that a step will be taken towards the metrication of charts in that soundings will appear in metres and, in conjunction with this, that tide tables will give the height of the tide in metres. This will be an unsatisfactory state since it is generally agreed that when a change of units is made it should be accomplished as quickly as possible. The present proposals will metricize the navigator in the vertical plane but leave him unchanged in the horizontal plane and in this half-converted state he will remain for years to come.

THE FuTuRE. To appreciate the changes required it is necessary to examine the various aspects of marine navigation.

For a long time to come the majority of merchant ships will probably continue to fix their mid-ocean position by astronomical sights. With this method of fixing the local vertical is the reference and determines latitude and longitude. It will obviously be convenient if these are retained as coordinates for stating position on the surface of the Earth but to be in strict accord with the system the units should be radians.

However, the latitude scale on a mercator chart will no longer serve as a distance scale. The S.I. unit of distance is the metre and hence a distance scale in metres will be required on every chart. On large-scale charts where the scale is effectively constant over the whole of the chart a metre scale placed in a convenient position will suffice: this is already provided on some. On smaller scale charts a continuously varying distance scale will be required and will be used in the same way that the latitude scale is used at present. For practical pur- 
poses distances will be measured in kilometres and speed in kilometres/hour and all instruments using the dimension of length will need re-scaling, e.g. distance and speed recorders, depth recorders and radar sets.

For great circle and rhumb line calculations the practical navigator assumes the Earth to be spherical and hence existing formulae will suffice with the addition of a conversion factor. For example if the length of $I^{\prime}$ on a spherical Earth is assumed to be $185^{2} \mathrm{~m}$. (the now obsolescent international nautical mile), then the rhumb line formulae become

where

$$
\begin{aligned}
\text { D. Lat. } & =(d \cos \theta) / 185^{2} \\
D . \text { Long. } & =(d \sin \theta \sec \phi) / 185^{2}
\end{aligned}
$$

$$
\begin{aligned}
& d=\text { distance in metres } \\
& \phi=\text { mean lat. } \\
& \theta=\text { rhumb line course }
\end{aligned}
$$

and $D$. lat. and $D$. long. are in minutes of arc.

The adoption of the radiation characteristics of the caesium atom as a measure of time interval will not have any noticeable effect on practical navigation but will cause some fundamental changes in the way that the navigator is taught.

No longer will orbital periods or the rotation of the Earth on its axis be measures of time. We no longer have to worry about the Sun as an erratic timekeeper or cope with the Mean Sun as a better one. In short we will not need to consider the equation of time and all the various 'years', 'days' and 'times' can be dispensed with. We merely acknowledge the fact that all the various celestial bodies and reference points have their individual rates of change of hour angle measured with reference to the one all-embracing atomic time.

Conclusion. It is evident that the adoption of S.I. units for navigation will lead to simplifications particularly when dealing with lengths. Those who will benefit most are the young people brought up on S.I. units who will learn their navigation without having to absorb many different units for the same dimension. Existing navigators may well prefer to remain unconverted but since the decision has been taken to adopt S.I. units it is best that the change over be made as quickly as possible.

There is evidently much scope for those who teach marine navigation to consider very carefully the future content and presentation of the subject.

BI B LI O G R A P HY

Changing to the metric system (1965). National Physical Laboratory.

\section{Hariot's Meridional Parts}

$$
\text { from Frank George }
$$

I was interested to see Mr. Pepper's note in the July issue of the Journal. The intriguing question of Hariot's unexplained approach to a problem that appears to require a knowledge of the integral calculus and exponential functions had 